\title{
Alexandre, vieux pote
}

Paul SAVOIE, poète

J'ai rencontré pour la première fois Alexandre Amprimoz dans les années 1970 lorsqu'il enseignait la littérature française à St. John's College, à l'Université du Manitoba. À ce momentlà, j'enseignais au Collège de Saint-Boniface, qui était affilié à la même université. Ce fut lors d'un colloque sur la littérature manitobaine où, ayant produit mon premier livre aux Éditions du blé, j'avais été un des auteurs invités. Même si notre rencontre fut brève, j'ai quand même reconnu chez lui la même passion devant la littérature et le même enthousiasme sans bornes pour la poésie. Il m'a alors confié qu'il était lui-même poète à ses heures et qu'il comptait un jour se faire publier.

Quelques années après cette première rencontre, Alexandre s'est mis à faire des recensions de mes livres. Il s'est alors permis de dire des choses très positives, très encourageantes face à mon écriture, ce qui m'a prédisposé à l'accueillir à bras ouverts dans mon cercle d'intimes. Mais notre amitié ne s'est pas concrétisée. Pendant plusieurs années, nos pas ont continué à se croiser de façon intermittente. À un moment donné, j'ai eu l'occasion de lui remettre la monnaie de la pièce et de faire une recension de Sur le damier des tombes, un recueil de poésie qu'il avait publié aux Éditions du blé au début des années 1980. Puis, à la même époque, Alexandre a communiqué avec moi pour m'inviter à participer à une rencontre littéraire à l'Université Brock, à Saint Catharines, où il était devenu professeur de littérature. C'est à partir de ce moment que notre amitié s'est concrétisée, autour du rapport que nous entretenions tous les deux avec la littérature en général, mais plus spécifiquement avec celle de nos milieux minoritaires respectifs. Alexandre se sentait aussi isolé comme poète à Saint Catharines que moi à Toronto. J'avais quand même un avantage sur lui, dans ce sens que, à Toronto, je côtoyais plus de poètes francophones qu'il ne pouvait le faire dans sa région. Cette conscience partagée de la fragilité de nos situations respectives et de la difficulté de faire rayonner nos œuvres nous a permis de créer un lien très fort et nous a aidé à forger une belle amitié à distance. Au cours des années 1980, nous avons souvent communiqué l'un avec l'autre, mais presque uniquement en ligne. Si nous étions très présents l'un à l'autre, c'était presque uniquement par le biais de nos rapports avec la littérature. Au cours de ces années, il ne nous est jamais arrivé d'aller prendre une bière ensemble, de fixer un rendez-vous dans un restaurant. Notre 
amitié se limitait aux échanges que nous pouvions avoir autour de ce qui était devenu notre point d'aimantation : 1'écriture.

Puis, à un moment donné, Alexandre a cessé d'écrire. Il a voulu se perfectionner dans le domaine de l'informatique. Il a quitté son poste universitaire afin de former sa propre compagnie. Nous nous sommes un peu perdus de vue.

Tout cela ne pouvait se terminer ainsi. Le destin en a voulu autrement. En 2009, au milieu de l'hiver, on m'a invité à donner un cours de création littéraire à l'Université Brock. C'était pour remplacer Alexandre, qui avait repris son ancien poste de prof de littérature, mais qui, atteint de diabète, avait dû prendre un congé de maladie en plein milieu du trimestre. La poésie avait encore fait des siennes et nous avait donné un moyen de renouer notre amitié. Cette fois, c'était de façon triste et indirecte, mais j'étais au moins revenu dans le champ d'activité de mon vieux pote qui, j'étais en train de l'apprendre, était bien apprécié par ses élèves et était connu sous le nom d'Alex. Pour moi, ce «vieux de la vieille », comme on se plait à nommer les vieux routiers comme lui et moi, allait toujours demeurer le même Alexandre, celui avec qui j'avais partagé ce même amour inébranlable pour la parole et l'expression poétiques.

Alexandre s'est remis de sa maladie et, lorsqu'il a repris ses cours, on m'avait confié d'autres cours à l'université, ce qui m'a permis de côtoyer cet homme à l'esprit fin, bien connu pour son sens d'humour imbattable. J'ai alors eu le plaisir, pour la première fois depuis notre longue et agréable association, de connaître la vraie personne en chair et en os. À plusieurs occasions, j'ai pu m'asseoir et jaser avec lui sur un tas de sujets, mais surtout sur l'importance du mot écrit et du besoin d'inventer des stratégies nous permettant d'inculquer chez les jeunes d'aujourd'hui ce même amour pour la littérature. Nous savions que notre défi était énorme. Mais nous savions également que c'était la chose qui nous unissait, lui et moi, qui occupait la place centrale dans nos vies. Et nous savions également que nous n'allions jamais abandonner la lutte. La poésie allait toujours demeurer le point de départ et le point d'arrivée de notre cheminement. Et, à cause de cela, notre amitié allait continuer à tout jamais à alimenter nos vies. 